\title{
How Enterprise must be Prepared to be “AI First”?
}

\author{
A pragmatic approach for AI adoption
}

\author{
Mustapha Lahlali ${ }^{1}$, Naoual Berbiche ${ }^{2}$, Jamila El Alami ${ }^{3}$ \\ Laboratory of Systems Analysis, Information Processing and Industrial Management \\ Higher School of Technology 11000- Salé, Mohammed V University, Rabat, Morocco
}

\begin{abstract}
Among disruptive technologies, Artificial Intelligence (AI), Robotic Process Automation (RPA) and Machine Learning (ML) play a very important role in Businesses Transformation and continues to show great promise for creating new sources of wealth and new business models. The reality of AI in the company is not reduced to a simple process optimization. In fact, AI introduces new organizational schemes, new ways of working, new optimization niches, new services, other ways of thinking about interactions with customers and therefore a new way of doing business. It thus reshuffles competitive data and imagine innovative processes to create new business models, offering new opportunities not only for IT solution providers but also for innovators, investors and business owners. Even if the contribution of Artificial Intelligence is not to be proved, many companies face difficulties in adopting this technology, mainly due to the lack of a pragmatic approach highlighting the roles and responsibilities of the various stakeholders, especially IT professionals and business owners and the key steps to follow to make this experience a real success. This research aims to answer fundamental questions, in particular: What will bring the implementation of this technology to the business of the company? How to prepare for this adoption? and if the decision to go is confirmed, what kind of adoption approach should companies follow? and finally how can Enterprises monitor this shift to the Intelligent edge.
\end{abstract}

Keywords-Artificial intelligence; machine learning; RPA; business transformation; AI adoption

\section{INTRODUCTION}

Artificial Intelligence was certainly born several decades ago, but its rhythm of adoption has clearly accelerated in recent years. Indeed, this technology is no longer reserved only for the scientific world and its rich, varied and innovative applications are a delight for many companies.

This acceleration was primarily driven by the explosion in the amount of data and the digital transformation, and also by the company's enthusiasm to adopt disruptive technologies in their growth strategy. Indeed, many companies, in order to remain competitive, have been forced to rethink the way they operate by integrating technological innovation into the daily process as an enabler for creating value.

Artificial Intelligence fully expresses its potential through various functionalities: it optimizes existing processes, automates routine tasks, allows assisted monitoring, detects abnormal phenomena, predicts future actions, and interacts more and more "naturally" with humans, thanks to recent developments in natural language processing.
This article aims to illustrate how AI can bring a tangible value to the enterprise by answering pragmatic questions. It shows, in a pragmatic approach, how companies can meet the challenges and takes benefits of adopting this technology by making the most of its contributions while minimizing the associated risks.

\section{ARTIFICIAL INTELLIGENCE IN BUSINESS: FoR WHAT PURPOSES?}

The development of Artificial Intelligence (AI) technology widens the limits of business practice for companies, thus promoting the transformation of information technology to optimize decision-making and operations. Recent research findings [1][2] presents several main developmental trends of the technology and the resulting challenges and show how dynamic AI capabilities can improve operational efficiency and business performance.

AI is definitively not a technological subject. It's, at the first level, a business matter aiming to improve the business performances and capabilities. Fig. 1 shows the main expectations of business managers towards the adoption of AI.

Artificial Intelligence can support three important major business needs: processes automation, cognitive signals, and cognitive engagement [4].

\section{A. Process Automation}

Robotic Process Automation paradigms (RPA) bring the opportunity to integrate robots inside the enterprise chain of value. RPA is not aiming to replace human, it's mainly designed to increase human productivity by automating repetitively and costly tasks [5]. Basically, RPA robots correspond to software programs acting as a digital worker and ensuring many kinds of tasks including:

- Automating customer relationship management and ensuring clear and comprehensive customer data collection and structuring.

- Ensuring that multiple data are correctly updating different systems, for example, blocking immediately customer identity in case of ID lost reclamation by customers.

- Verifying the integrity of data manipulated in different systems to ensure the authenticity of information managed in different business lines, for example, verifying automatically inside the billing system, the amount of a settlement check provided inside the supplier bills. 


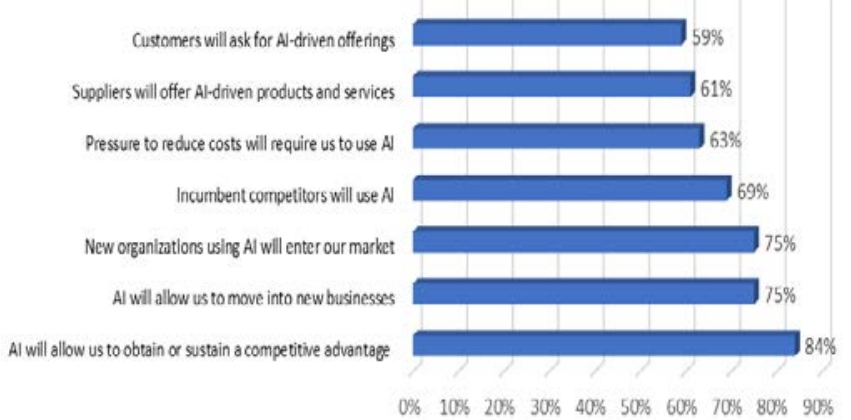

Fig. 1. Reasons for Adopting AI [3].

RPA is usually considered as the least expensive and easiest to implement module of the cognitive technologies and typically brings a quick and high return on investment.

Some enterprises launched RPA pilots in accounts payable, automatic settlement check controlling and validation, automation of IT daily tasks by avoiding manual operations and minimizing human intervention.

In all cases, RPA is not a Human predator [6]. It's a new way to let Human making valuable tasks and being supported by robots to do well and correctly daily tasks. The cost killing objective is usually forecasted by enterprises beside the use or not of the RPA. For this reason, the outsourcing/offshoring sector will be challenged by this paradigm and maybe the job losses impact can be felt more in those areas [7].

\section{B. Cognitive Signals}

Cognitive signals can detect patterns in vast volumes of data and interpret their meaning (e.g., predicting purchasing habits, identifying credit card frauds, determining trends, qualifying data quality,...).

Cognitive signals provided by Machine Learning are stronger and more sophisticated than those provided by analytics for those three principal reasons:

- The huge amount of data.

- The ability for Machine Learning-based systems to learn from the data collected.

- The prediction capabilities to anticipate features.

It should also be noted that Machine Learning bring new capabilities and features like text and voice recognition. In the past, a lot of efforts had to be made to collect and to manipulate structured data. Today, Machine learning brings a significant contribution to do these tasks more intelligently for both structured and unstructured data thanks to the important developments in computing power.

Cognitive signal applications are typically used to improve performance on jobs and tasks only machines can do, so we can therefore imagine a fruitful collaboration between humans and machines.

\section{Cognitive Engagement}

Smart assistants are revolutionizing the daily lives of individuals and businesses. With their ability to interpret and understand human language through Machine Learning capabilities, they enable the following opportunities:

- Intelligent agents [8] ensuring customer assistance every day by supporting simple to complex queries via quick and concise responses.

- The product offering is enhanced by learning from the various customer questions addressed to chatbots, allowing the company to anticipate customer demands and to differentiate itself from the competition.

- Internally, the distance between the company and its employees is reduced by setting up intelligent channels of exchange and support for daily queries (e.g., Administrative HR assistance, IT help desk,...).

Fig. 2 shows the different types of AI adoption within Companies. It gives a clear visibility on the current use and forecasts of the future uses of the intelligent technologies on which the $\mathrm{AI}$ is based to transform the business processes.

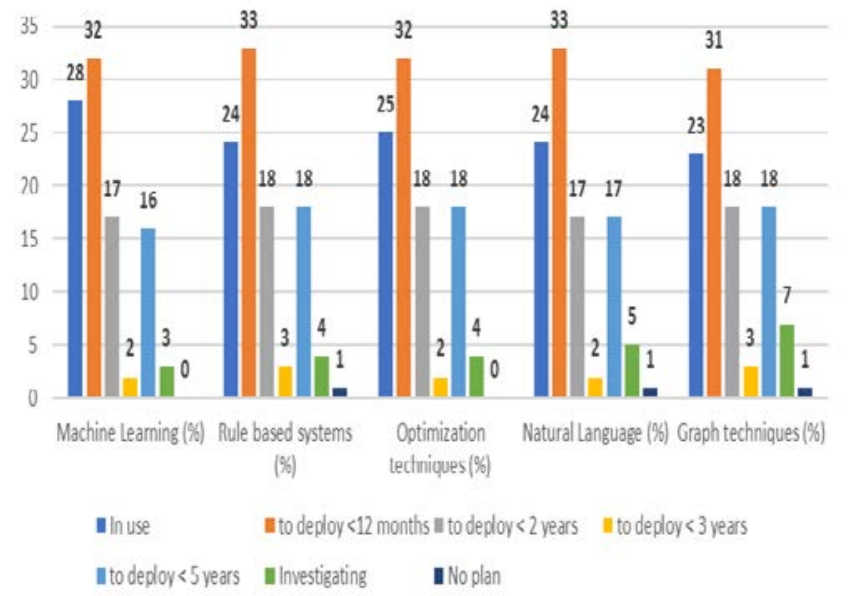

Fig. 2. AI Adoption by Type [9].

\section{Challenges Facing AI Integration AND Adoption}

Companies must put in place the necessary prerequisites for the success of its AI integration projects, which are related, in particular, to technological, financial, organizational and human aspects.

It should be noted that once the technology adoption process is successful, the impact on the business can be very important. For example, in the retail industry, a lot of champions made a significative growth by adopting IA like Amazon and Walmart [10].

\section{A. Understanding the Technologies}

Before going in depth and embarking on AI adventures, companies must ensure that they have a thorough understanding of the technology and be able to make a distinction between different paradigms and their potential for value creation. For example, RPA offers no ability intelligence or learning, it automates the cumbersome and repetitive tasks, unlike the deep learning where there is a strong element of intelligence. In all cases, AI-driven companies will have the potential to compete by looking beyond the initial product or service to the full potential of the technology available [11]. 
From talent management perspective, companies must prepare task forces which are necessary to manage these AI projects. From Data Scientist to AI Architect, each role has a specific mission to leverage one or multiple sides of the technology in order to succeed in AI projects. Unfortunately, this is not enough. The construction of an ecosystem made up of experts and industrialized product offerings is considered as a fundamental pillar in the mastery of technology and a guarantee to ensure its viability.

\section{B. Financial Effort}

Experimentation of the Machine Learning and Data Intelligence subjects require a significant financial effort which must be justified from the point of view of viability. In fact, to make this successful, companies are called upon to mobilize a lot of expertise which requires large expenses. The whole challenge lies in the adequacy between the expenditure and the return on investment which is generally difficult to ensure given the high level of uncertainty that characterizes a large number of AI projects.

Also, thanks to the learning techniques offered by AI (e.g. supervised, unsupervised, by reinforcement) [12] [13], many opportunities are now available to companies to create new business models and offer a new form of value. In this context, the predictive aspect remains an undoubtedly promising axis for designing intelligent systems capable of reasoning, analyzing and perhaps making decisions.

Some questions will arise in terms of risk management accompanying this revolution, but from a business point of view, several possibilities arise for switching approaches from classic IT solutions towards intelligent and augmented solutions.

Faced with increased competition, IT Departments will find themselves at the center of all interests and will transform from a support unit to a profit unit because in the era of intelligence, there mission will not be to support the trades but to transform the whole operational model of the company by making intelligence an axis of wealth. In this perspective, IT teams will find themselves in a new management style of collaborative work and agile implementation with a record speed of change and transformation and also with a drastic impact on the product development methodology and the implementation of intelligent journeys.

It is almost certain that the "Augmented CIO (Chief Information Officer)" is a CIO who will use "intelligence" as a workhorse to enhance its contribution to the company's strategy.

\section{Organization Challenges: Internal and External Dependencies}

Faced with the diversity of profiles and the complexity of AI subjects, many organizational challenges will be the object of challenge. Some companies have adopted the Chief AI Officer (CAIO) function as an unifying role to create synergy between business, IT and statisticians and make teamwork more fluid and more constructive.
Most AI topics are expert and advanced computing topics which require the collaboration of several profiles from different structures, whether internal to the company or located in its ecosystem (e.g. service providers, partners, IT experts). In this situation, managing relationships between different teams is almost considered as a daily challenge. For example, in terms of big player dependencies, many suppliers are positioning themselves in this segment without necessarily having a solid foundation with commercial promises that are not necessarily tenable. For this reason, many companies consider that Open source-based solutions are an issue that will not depend on the big players, but at the same time it requires the loyalty of internal experts which is not at all an easy task. In this perspective, the real challenge will consist in finding a balance between business ambition, technological capacity and a better organization to co-construct intelligent value.

\section{Thinking Platforms}

AI is drastically transforming the technological DNA of the company. In fact, the rate of dependence on experts and IT giants remains too high. In this sense, the company must adopt a new approach. The first one is based on internal Labs to develop and retain its own internal experts by associating them with the company's businesses. The second point concerns the implementation approach and methodology. In fact, supplying the process of creating AI products is totally different from traditional IT products, hence from a technical standpoint, the company must adopt a reasoning based on platforms and not on isolated solutions. These platforms will aim to industrialize the entire chain from data collection to the operationalization of machine learning models with the possibility of mixing between the private cloud and the public cloud to optimize production capacities and have a very significative return on investment.

\section{E. Skills and Task Forces}

Companies that want to win the race for a successful AI adoption need to be truly convinced that this victory comes first and foremost through the provision of skills, competencies and talents. Furthermore, developing and maintaining smart talents is a very difficult HR subject because it's about innovation in HR management practices to create suitable policies to smart task forces highly targeted by competitors. Enterprise should protect their internal skills by supporting them and rewarding their efforts.

The availability of qualified resources is a precondition for a successful leap towards the era of intelligence. Different modes of organization are possible such as internal competence centers and Tech accelerators. The intangible capital of the company represented in its human wealth is definitely a sure catalyst for success.

The sheer diversity and complexity of AI projects combined with a requirement for rapid time to production create the need to find key AI roles to achieve successful AI projects. Fig. 3 shows main AI specialist's roles and responsibilities [14]. 


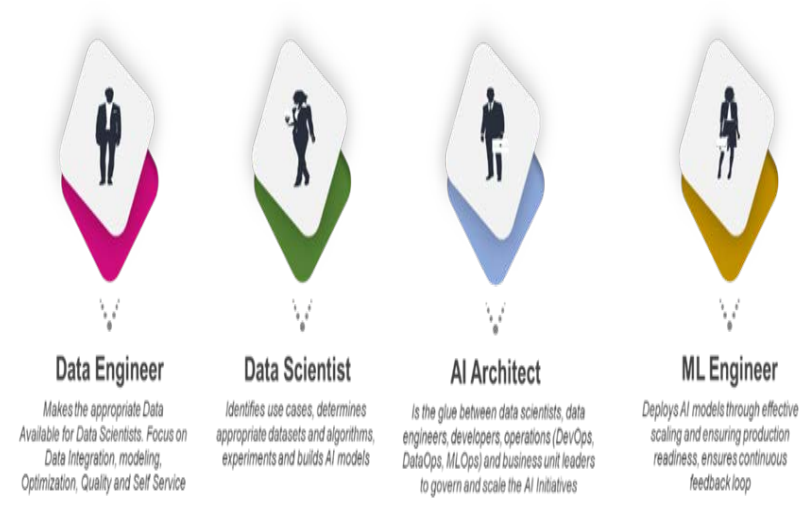

Fig. 3. AI's Core Roles and Responsibilities.

\section{F. Ethics}

A question that comes up quite often in AI implementation projects concerns the limitation of ethical responsibilities for decisions that might be made by an algorithm based on machine intelligence [15].

For example, administrative, social or financial decisions can be subject to a complaint. Indeed, the decisions taken by human can consider emotional et behaviors factors, even subjective in some cases, that a machine cannot necessarily make. All the effort therefore remains to clearly define the tolerance thresholds and to delimit responsibilities between human and machines.

\section{AI Initiatives And Projects: The Way of Success}

The success of an initiative based on AI begins with the identification of opportunities, the determination of the use cases to better achieve these opportunities and finally the choice of the right technology and solutions to meet the expected results.

\section{A. Creating a Portfolio of Projects and Identifying Opportunities}

AI programs [16] are becoming more and more present in companies' IT portfolios. These programs consist of a several use cases and projects generated, most of the time, after an experimentation phase. This structuring approach helps the company to choose and prioritize AI initiatives and projects according to business needs, market opportunities and needs, team capacity and also taking in account prioritization of intelligence technologies according to the context and providers maturity. The management of these programs allows top management to continuously monitor the evolution of the company's intelligence quotient.

\section{B. Targeting the use Cases}

To approach AI projects, companies must start with an exploration phase which consists in identifying concrete use cases where intelligence is essential and also which constitute a real axis of improvement and wealth creation.

These use cases will be essentially inspired by the strategy of the company, its operational objectives, the market opportunities and, above all, the trajectories identified to ensure growth.
Basically, the use cases must be proposed and especially well defined in feasibility term correlated to the expected value. The result of this work will offer the company a measured trajectory to appropriate the technology and move slowly but surely towards the intelligent enterprise.

\section{Selecting Tools and the Technology}

One of the major challenges to be faced is to properly identify the right technology through which intelligence will be showcased.

For example, chatbots or digital assistants can be used in certain contexts where the company must be very close to its customers and respond in record time to their requests. As for facial recognition, which is very data-intensive, it is of interest to use in contexts of identification or detection of abnormal behavior. In all cases, AI architects must use the right channel to better achieve the desired impact.

\section{THREE STEPS TO INTEGRATE AI IN ENTERPRISE BUSINESS APPLICATIONS}

Once AI-based initiatives are successful, companies can move up to integrate these use cases into their process. To achieve this objective, companies must first launch pilot projects to clearly identify all aspects related to the implementation of the technology (technical prerequisites, financial impacts, risks, organizational aspects, Human Resources, impact on the Ecosystem,...). The second step relates to the optimization, review and redesign of the processes. The third and last phase obviously relates to the full-scale generalization.

\section{A. Setting up Pilot Projets}

The creative appetite for intelligent products and services is always faced with the contradiction between promises and real creative capabilities. For this reason, companies from the Test \& learn perspective, must test as part of Proof of Concepts (PoCs) the Business impact on a reduced scope before any full-scale realization.

The SENSE-THINK-ACT [17] model can be a suitable tool to challenge AI PoCs, by preparing Data, designing products and creating AI powered solutions.

The phase of testing and validating the added value of the technology on the business processes remains a key stage in AI adoption. Companies must in no case derail the rule under the pretext of submitting to external pressure or simply following the fashion effect.

\section{B. Redesigning Processes}

The implementation of any projects based on Artificial Intelligence does not mean necessarily the fact that the company should automates its processes in an iso functional way. To get the most out of the technology, companies must think about reshaping their way of doing things (thinking out of the box), so that machines and Humans can perform in perfect harmony, because, at the end, each actor should compensate the weaknesses of the other.

Hironori Takeuchia and Shuichiro Yamamoto [18] worked on a Generic business-AI alignment model giving the capacity 
to integrate AI within the Enterprise architecture and giving a strong and didactic way to facilitate AI integration with business processes.

Today, every business realizes the drastic impact of enterprise architecture on the leap to intelligent enterprise. This integration is illustrated in Fig. 4 according to the Enterprise AI Canvas [19].

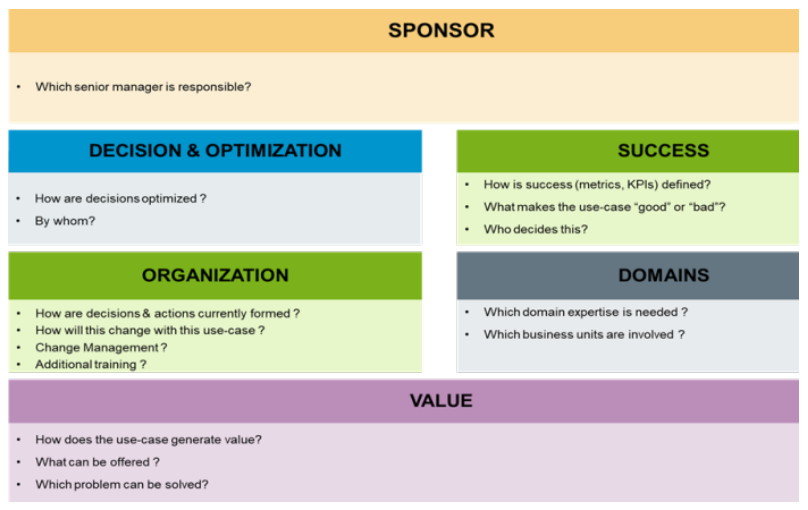

Fig. 4. Enterprise AI Canvas.

Working in a common framework under solid governance is far from being a choice. Indeed, the orchestration of flows and the orchestration of exchanges is necessary to successfully integrate AI into the company and ensure that all professionals and AI engineers work in perfect synergy.

\section{Scaling Up}

Several experiences have shown that many companies having passed "successfully" proof of concepts stage, have unfortunately failed to make it a real use case, because the mastery of technology and IT talents cannot be considered as the only factors guaranteeing success [20].

Indeed, before any generalization of an experimentation, companies must put in place the necessary prerequisites for its success, in particular: a perfect synergy and convergence of point of view and vision between the business owners and the IT professionals, an adequate governance framework and a solid change management to allow the appropriate use of the future system.

The Scaling up approach is a mix between the AI adoption degree and the knowledge of the technology as shown in Fig. 5:

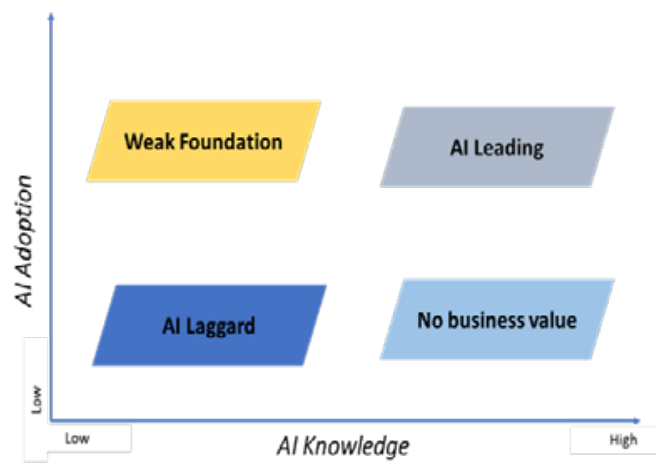

Fig. 5. Blueprint for AI Scaling up.

\section{CONCLUSION}

Today, no one can deny the importance and the contribution of Artificial Intelligence in the transformation of our way of acting, driving our processes and especially of creating value. To take maximum benefits from this technology, companies must prepare the prerequisites for a successful adoption, in terms of understanding the technology, coordinating initiatives, making data available and implementing innovative use cases.

In that way, to make the implementation of AI projects a happy story, company faces many challenges and it is brought to change management issues due to the massive adoption of disruptive technologies, the use of structured and unstructured data and the transformation in the ways of doing business.

Our work aims to implement a Model of AI adoption inside the Enterprise. We call it 2PS Model (Pilot, Project and Scale) giving the opportunity to leverage AI adoption by implementing holistic approach. Each step must be well prepared and well executed to ensure success.

Within this study, we tried to answer a couple of questions regarding enterprise readiness to Adopt AI. At this stage, aspect concerning Explainable AI and Trust AI are not discussed in this paper. In future publication we will challenge those aspects as perspective and evolution of our thinking about this problematic.

Our approach can be compared with some frameworks like Google AI readiness framework which deal with AI adoption from Learn, Lead \& Access perspective by challenging tactical, strategic and transformation aspects. We deal with the AI adoption from strategic point of view to give the core capacities to enterprise to deal with AI disruption. The Google framework supposes that enterprise is smart enough to go further in its AI journey. In that way, our work aims to prepare the enterprise to be able to leverage frameworks (like google or other) to create maximum value for user experience and a better contribution for enterprise growth.

Finally, AI platforms are basically based on Data, Machine Learning and Models Operations. So, as another perspective of this research, we aim to analyze how to leverage those capabilities to give a strong enabler for AI adoption through the implementation of a case study to illustrate the disruptive impact of the AI on business process. We aim within the future work demonstrate the power of this technology, particularly these aspects related to Data Science and Machine Learning, on improving both the internal performance of the company and its interactions with its eco-system.

\section{REFERENCES}

[1] Y. Chen, z. Lin: "Business intelligence capabilities and firm performance: a study in china”, International journal of information management, August 2020, p. 4.

[2] S. m. Correia loureiro, j. Guerreiro, l. Tussyadiah: “Artificial intelligence in business: state of the art and future research agenda”, Journal of business research, November 2020.

[3] S. Ransbotham, d. Kiron, p. Gerbert, m. Reeves: "Reshaping business with artificial intelligence closing the gap between ambition and action", MIT sloan management review, October 2019, p. 5.

[4] T. H. Davenport, R. Ronanki : “On AI, Analytics, and the New Machine Age”, Harvard business review, 2018, p 11. 
[5] J. G. Enríquez, a. Jiménez-ramírez, f. J. Domínguez-mayo, j. A. Garcíagarcía: "Robotic process automation: a scientific and industrial systematic mapping study”, Ieee access, March 2020, pp 1-4.

[6] Wil m. p. Van der Aalst, m. Bichler, a. Heinzl: "Robotic Process Automation”, Springer, May 2018, p 2.

[7] J. Surowiecki: “The great tech panic: Robots won't take all our jobs”, www.wired.com, 2018.

[8] T. Boobier: "AI and the future of banking”, Wiley finance series, May 2020, pp 261-265.

[9] S. Sicular: "The hype cycle for artificial intelligence 2020 reflects the state of $\mathrm{AI}$ in the enterprise", Gartner, October 2020, p. 1.

[10] F. D. Weber, r. Schütte: "State-of-the-art and adoption of artificial intelligence in retailing”, Digital Policy, Regulation and Governance, 2019, p. 9.

[11] K. Lakhani, m. Lansiti: "Competing in the age of AI: strategy and leadership when algorithms and networks run the world", Harvard business review, January 2020, pp 8-10.

[12] Cigref: "Artificial intelligence in companies: strategies, governance and data intelligence challenges”, October 2018, p. 4.
[13] R. e. Neapolitan, x. Jiang: "Artificial Intelligence, With an Introduction to Machine Learning, SECOND EDITION”, CRC Press, 2018, PP 89 - 330.

[14] L. Goasduff: "How to staff your AI team”, Gartner, November 2020, p.2.

[15] B. W. Wirtz, J. C. Weyerer, C. Geyer: "Artificial Intelligence and the Public Sector-Applications and Challenges", International Journal of Public Administration, 2018, p. 11.

[16] E. Brynjolfsson, a. Mcafee: "The business of artificial intelligence", Harvard business review, July 2017.

[17] N. Soni, e. K. Sharma, n. Singh, a. Kapoor: "Impact of artificial intelligence on businesses: from research, innovation, market deployment to future shifts in business models", International conference on computational intelligence and data science (iccids), April 2019, p. 8.

[18] H. Takeuchi, s. Yamamoto: "Business analysis method for constructing business-AI alignment model”, 24th International conference on knowledge-based and intelligent information \& engineering systems, 2020, pp 4-9.

[19] U. Kerzel: "Enterprise AI canvas integrating artificial intelligence into business”, Arxiv, September 2020, pp. 10-11.

[20] B. Mccarthy, s. Tamim: "Building AI powered organization”, harvard business review, August 2019, pp 9-13. 